\title{
Similarity of Jupiter and RRATs
}

\author{
Ilknur Gezer and E. Rennan Pekünlü \\ Department of Astronomy and Space Sciences, Faculty of Science, University of Ege, Bornova, \\ 35100, Izmir, Turkey \\ email: gezer.ilknur@gmail.com \\ email: rennan.pekunlu@ege.edu.tr
}

\begin{abstract}
In the present investigation, radial diffusion of equatorially trapped electrons in the magnetospheres of Jupiter and Rotating Radio Transients (RRATs) are examined and compared. It is assumed that electrons lose energy through synchrotron radiation and the wave-particle interaction. The phase space density of the electrons, which go through gradB drift in Jupiter's and RRATs magnetospheres and thus resonate with the plasma waves, changes and this change predicted by the model seems to be consistent with the Pioneer 10 and Pioneer 11 data for Jupiter's case and a similar result obtained for RRATs.
\end{abstract}

Keywords. RRATs, Jupiter, radio bursts, wave-particle interaction

\section{Introduction}

Parker Multi beam Survey began in August 1997 and was completed in March 2002 and over 700 new pulsars were discovered. 11 of these pulsars are previously unknown type of sources. These sources have been described as a Rotating Radio Transients. They show single, dispersed burst having durations within the range of 2-30 ms. Maximum flux density of radio bursts are $\sim 0.1-4$ Jy and the average time intervals between bursts range from 4 minutes to 3 hours. Periodicities 10 of the 11 sources are in the range $0.4-7 \mathrm{~s}$. Period variations were measured for 3 of these 11 sources, through the measurements of magnetic fields $\left(10^{12}-10^{14} \mathrm{G}\right)$ and ages (0.1 $\left.3 \mathrm{My}\right)$ (M.A. McLaughlin, 2006). Nature of these sources is not known yet, but it is assumed that the sources which show different radio properties in comparison with known radio sources, are field neutron stars of our galaxy.

Luo and Melrose (2007) have proposed that radiation belts that are similar to the planetary magnetosphere may exist for a pulsar which have relatively long period and a strong magnetic field. Polar cap model does not explain observed intense radio burst of these sources because most of RRATs have long periods and pair production is not effective. Similarity of Jupiter and pulsars magnetospheres are known subject for a long time (e.g. de Pater, 2004, Hill, 1995). Luo and Melrose (2007) have suggested that pulsars may have radiation belts in the closed field lines region where relativistic plasma are trapped. The trapped region is believed to be so similar to the Earth's radiation belts. The same authors suggest that low-frequency waves occurring in pulsar magnetosphere can disrupt the trapped plasma and it can lead to intense precipitation toward the neutron star surface. Intense radio bursts are thought to be produced by these precipitating particles toward the star's surface. Equatorially trapped particles while radially diffusing towards the surface of RRATs at the same time may resonate with the plasma waves and be raised to relativistic energies. In situ measurements showed that in van Allen radiation belts electrons are raised by this process to energies as high as $10 \mathrm{MeV}$ (Home, 2005). These energetic particles then precipitate towards the stellar surface and may initiate the intense radio bursts as proposed by Lou and Melrose (2007). 
Radial diffusion of equatorially trapped particles in Jupiter's magnetosphere is examined by Pekünlü $(1992,1995)$. The author assumed that the first and the second adiabatic invariants are conserved and the third one is violated. Pekünlü $(1992,1995)$ also assumed that particles lose energy with synchrotron radiation and wave particle interactions. In this respect, the mechanism proposed by Luo and Melrose (2007) to explain unusual radiation properties of RRATs is similar to that occurring during radial diffusion in Jupiter's magnetosphere. In this investigation we try to apply the method Pekünlü(1992, 1995) used in his early study to RRATs.

\section{Radial Diffusion Mechanism}

Generally there are four processes in the radiation belts of planets magnetosphere. These are:(i) injection of charged particles into the trapping region of the magnetosphere from the solar wind through the day side neutral points, (ii) acceleration, (iii)diffusion,(iv) loss (Schulz and Lanzerotti, 1974).Observation by Simpson et al. (1974) and Simpson (1974) with Pioneer 10 have shown that radial diffusion is the main process in radiation belts. The type of radial diffusion that conserves both magnetic moment $\mu$ (the first adiabatic invariant) and flux density $\mathrm{J}$ (the second adiabatic invariant).Convenient conditions for both $\mu$ and $\mathrm{J}$ is conserved case are magnetic sudden impulses and other magnetic disturbances operating on the same time scale. Actually there are three motion in the magnetosphere; longitudinal drift, bounce motion and cyclotron motion. Their time scales are given by, $\tau_{D} \geqslant \Delta t \geqslant \tau_{b} \geqslant \tau_{c}$ and $\Delta t$ where drift period,bounce period,cyclotron period and period of disturbance.Radial diffusion mechanisms which $\mu$ and/or $\mathrm{J}$ is not conserved processes are less important than sudden impulses in radiation belt dynamics since particles can not be energized efficiently in the process.(Pekünlü,1992).Pekünlü has indicated in the same paper that radial diffusion requires that at least the third invariant should change.Since the period of the drift in longitude is the longest of the three periods mentioned above.Random variations of potential electric fields can cause violation of the third invariant and thus lead to radial diffusion.(Roederer, 1970).Radial diffusion formula is given by(Pekünlü,1992)

$$
\begin{aligned}
D_{o} L^{n} \frac{\partial^{2} f}{\partial L^{2}}+(n+2) D_{o} L^{n-1} \frac{\partial f}{\partial L} & +\left\{\frac{2(n-1) D_{o}}{L^{2-n}}+\frac{\beta B_{0}^{2}}{m_{0} c^{2} L^{6}}\left[3\left(1+\frac{2 \mu B_{0}}{m_{0} c^{2} L^{3}}\right)^{1 / 2}\right]\right\} f \\
& +\frac{\beta B_{0}}{L^{3}}\left(1+\frac{2 \mu B_{0}}{m_{0} c^{2} L^{3}}\right)\left[\left(1+\frac{2 \mu B_{0}}{m_{0} c^{2} L^{3}}\right)^{1 / 2}-2\right] \frac{\partial f}{\partial \mu}=0
\end{aligned}
$$

where $f(\mu, J, L, t)$ represents the particle distribution function, $\mathrm{L}$ is McIlwain's magnetic shell parameter $D_{L L}$ is the diffusion coefficient. $L=R / R_{j}, B=B_{0} L^{-3}, B_{0}$ is the equatorial surface field strength, $D_{L L}=D_{0} L^{n}$ is the radial diffusion coefficient where $D_{0}$ is the non-radial dependence and is calculated from the Pioneer data as having a value $D_{0} \sim 2.5 \times 10^{-6} R_{j}^{2} s^{-1}$ and $\mathrm{n}$ indicating the power dependence on radial position. $\mathrm{n}=2$ value have been used since it provides a very good fit to Pioneer 10 data. Finally the steady state assumption, $\partial \mathrm{f} / \partial \mathrm{t}=0$, have been used (Pekünlü,1992). In the same paper Pekünlü have calculated that the numerical integration of radial diffusion equation for Jupiter over the interval $\mathrm{L}=1$ to $\mathrm{L}=5$, using Gaussian elimination method.

In the present investigation,radiation processes in the magnetosphere of RRATs', as described by Rotating Radio Transients and of Jupiter are compared. Radial diffusion of electrons trapped in the magnetic equator of Jupiter and RRATs is explored. A similar analysis, which has been done for Jupiter, was carried out for RRATs. As a result of the exploration we have obtained the above result. 


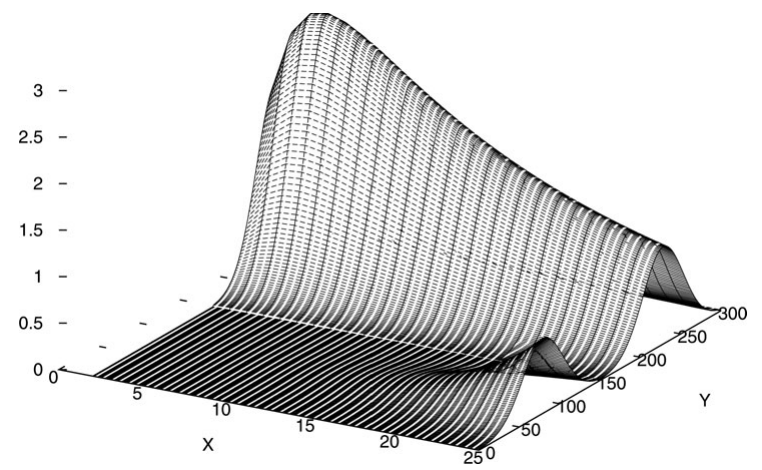

Figure 1. Jupiter's case; If the electrons lose energy only synchrotron radiation, f phase space distribution is in range $0 \leqslant Y \leqslant 150$. In the fig. $1 \mathrm{x}$ - direction is $\mathrm{L}$ and $\mathrm{y}$ - direction is $\mu$. If the electrons lose energy both synchrotron radiation and the wave-particle interaction, $f$ phase space distribution is in range $150 \leqslant \mathrm{Y} \leqslant 300$. Fig. 1 has been drawn for $212 \mathrm{MeV} / \mathrm{G}$.

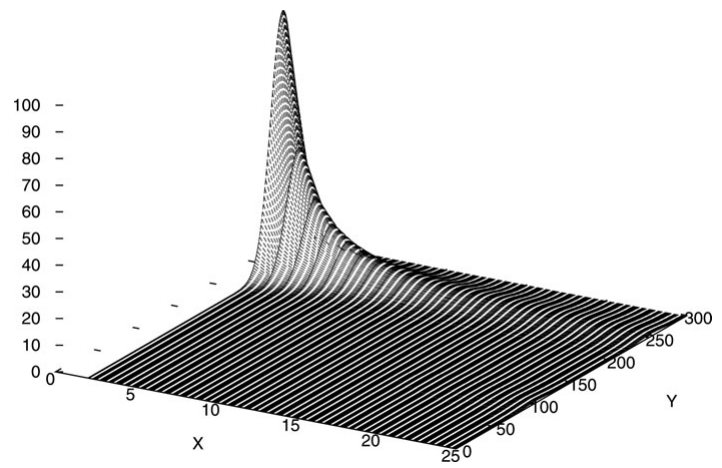

Figure 2. RRATs's case; Fig.2 has been drawn for calculated three RRATs'average magnetic moment value which is $330 \mathrm{MeV} / \mathrm{G}$.

\section{Results}

In this examination we considered only the radial diffusion. Fig. 2 shows that the phasespace density of electrons diffusing radially inward increases toward the surface of a RRATs. This enhancement in the number of electrons may be the reason for the coherent emission.

In the present study we could not work out the time scale for bursts, neither the flux density of the radiation to see if the radial diffusion could explain the observed properties of the radio bursts. Second shortcoming of the present study is the uncertainity in the diffusion coefficient, $D_{L L}$ for RRATs.

If the burst we receive from RRATs are generated by streaming instability caused by downward moving particles along the magnetic field lines, as proposed by Lou and Melrose (2007) then both the processes of radial diffusion and the pitch-angle diffusion should be taken into account.

\section{References}

Becker, W. 2009 Springer-Verlag Berlin Heidelberg, Neutron Stars and Pulsars, p. 41 de Pater, I., Kassim, N. \& ve Rucker, H. O. 2004, Planetary and Space Science,15, 1339-1341 Hill, W. T. ve Dessler, A. J 1995, Earth in Space, 8, No.2, 6.

Horne, R. B. 2005, Nature, 437, 227 
Luo, Q. \& Melrose, D. 2007, MNRAS,378, 1481L

Mc Laughlin 2006, Nature, 439, 817M

Pekunlu, E. R. 1992, Earth, Moon and Planets, 59, 201

Pekunlu, E. R. 1995, Earth, Moon and Planets

Popov, S. B., Turolla, R., \& Possenti, A. 2006, MNRAS, 369, L23-L26

Shannon, R. \& Cordes, J. M. 2006, AAS, 20915910S 